Review

\title{
The functional roles of long noncoding RNA DANCR in Human Cancers
}

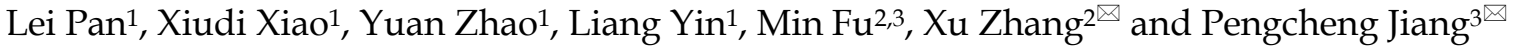 \\ 1. Department of Breast Surgery, The Affiliated People's Hospital of Jiangsu University, 8 Dianli Road, Zhenjiang, Jiangsu 212002, China. \\ 2. Jiangsu Key Laboratory of Medical Science and Laboratory Medicine, School of Medicine, Jiangsu University, 301 Xuefu Road, Zhenjiang, Jiangsu 212013, \\ China. \\ 3. Department of General Surgery, The Affiliated People's Hospital of Jiangsu University, 8 Dianli Road, Zhenjiang, Jiangsu 212002, China. \\ $\triangle$ Corresponding authors: Xu Zhang, Jiangsu Key Laboratory of Medical Science and Laboratory Medicine, Jiangsu University. 301 Xuefu Road, Zhenjiang, \\ Jiangsu 212013, China. E-mail: xuzhang@ujs.edu.cn; Pengcheng Jiang, Department of General Surgery, The Affiliated People's Hospital of Jiangsu University. 8 \\ Dianli Road, Zhenjiang, Jiangsu 212002, China. E-mail: pc_jiang@126.com.
}

(C) The author(s). This is an open access article distributed under the terms of the Creative Commons Attribution License (https://creativecommons.org/licenses/by/4.0/). See http://ivyspring.com/terms for full terms and conditions.

Received: 2020.01.30; Accepted: 2020.07.20; Published: 2020.10.12

\begin{abstract}
Long noncoding RNAs (IncRNAs) have been wildly explored in various cellular processes and their aberrant expression could lead to tumorigenesis, development and progression. Differentiation antagonizing non-protein coding RNA (DANCR), a well-known IncRNA that is aberrant expression in various tumors, including hepatocellular carcinoma, gastric cancer, colorectal cancer, breast cancer, lung cancer and glioma and so on, in which it functions as oncogene mainly, contributing to cancer development and progression. High expressed DANCR is correlated with poor prognosis. In the present review, we summarize recent progression concerning the role, potential clinical utilities and underlying molecular mechanisms of DANCR related to occurrence and development of multiple cancers.
\end{abstract}

Key words: long noncoding RNA, DANCR, biomarker, therapeutic target

\section{Introduction}

Cancer is fundamentally caused by genetic alterations that result in the deregulation of the cellular information flow to alter cellular homeostasis and promote growth $[1,2]$. Most cancers occur as a result of somatic interaction and germline mutations with a variety of environmental factors. And many of these mutations are in regions of the genome that lack protein-coding capacity, yet contain other type of genes that act as RNA molecules, such as the noncoding RNAs [2].

Long noncoding RNAs (lncRNAs) are defined as transcripts of more than 200 nucleotides without obvious protein coding potential [3]. The majority of lncRNAs are transcribed by RNA polymerase II, and are capped and polyadenylated at the $5^{\prime}$ and $3^{\prime}$ ends, respectively [2]. Increasing evidence has demonstrated that lncRNAs have tissue-specific expression and are expressed in a regulated manner in various cancers [4]. In addition, lncRNAs influence cell cycle regulation, survival, immune response or pluripotency, and other functions in correlation with distinct gene sets, which determine the transformed phenotype of cancer cells [4]. Furthermore, several lncRNAs are transcriptionally regulated by key tumor suppressors or oncogenes [2]. For example, lncRNA metastasis-associated lung adenocarcinoma transcript 1 (MALAT1) is overexpressed in various tumors, associated with metastasis and invasion, and promotes progression of hepatocellular carcinoma, osteosarcoma and bladder cancer [5-7]. In contrast, NF-KB interacting lncRNA (NKILA) is a tumor suppressor and acts as a NF-kB regulator to inhibit breast cancer metastasis [8-10]. Taken together, IncRNAs are functional transcripts that contribute to the hallmarks of cancers, and therefore becoming attractive potential therapeutic targets [11-13].

LncRNA differentiation antagonizing nonprotein coding RNA (DANCR) also termed as antidifferentiation non-coding RNA (ANCR), which is originally identified a regulator of the suppression of 
progenitor differentiation [14]. Subsequent studies demonstrated that DANCR is overexpressed in many human malignancies, including lung, liver, colon, gastric and breast cancers. In addition, DANCR has been implicated in regulating cancer cell proliferation, apoptosis, migration and invasion and be associated with poor prognosis. This review summarizes the current studies of functions and regulation mechanisms of DANCR in human cancers.

\section{Discovery of DANCR}

In 2011, Kretz et al. discovered an uncharacterized, predicted lncRNA, NR_024031, termed ANCR, was suppressed upon terminal differentiation of multiple cell types [14]. The ANCR is an 855-nucleotide lncRNA and its gene locates at chromosome 4, with the closest adjacent annotated genes located $54.8 \mathrm{~kb}$ upstream of USP46 and $28.7 \mathrm{~kb}$ downstream from ERVMER34-1 oncogene. The ANCR locus consists of three exons and harbors MIR4449 and SNORA26 in introns 1 and 2, respectively [14]. Recent studies have demonstrated that DANCR was overexpressed in major types of cancers, and its expression was closely associated with clinicopathological characteristics, including lymphatic metastasis, distant metastasis and tumornode-metastasis (TNM) stage. Current evidences suggested that DANCR was associated with important cellular mechanisms and functioned as tumorigenesis, colonization, tumor invasion, metastasis, proliferation, migration, apoptosis, disease progression and prognosis in numerous cancers. The relevant clinicopathological features and underlying molecular mechanisms of DANCR in various cancers are summarized in Tables $\mathbf{1}$ and $\mathbf{3}$.

\section{DANCR in various cancers}

Accumulating evidences have demonstrated the abnormal expression of DANCR in multiple cancers, including hepatocellular carcinoma, gastric cancer, colorectal cancer, pancreatic cancer, breast cancer, lung cancer, glioma, osteosarcoma, cervical cancer, bladder cancer, prostate cancer, nasopharyngeal carcinoma, esophageal squamous cell carcinoma, ovarian cancer and retinoblastoma. The associations between DANCR expression and the relevant clinicopathological features of various cancers are summarized in Table 1 . And the potential as biomarkers for diagnosis and prognosis of DANCR in cancers are presented in Table 2. The functions and underlying molecular mechanisms of DANCR in various cancers are summarized in Table 3 and detailed in the rest of this review.

\section{Hepatocellular carcinoma}

Yuan et al. found that DANCR was overexpressed in stem-like hepatocellular carcinoma cells and associated with CTNNB1, which could block the repressing effect of miR-214, miR-320a and miR-199a [15]. And DANCR was up-regulated in tumor tissues and plasma of patients with hepatocellular carcinoma, and plasma DANCR might be a useful biomarker for hepatocellular carcinoma diagnosis. DANCR expression was highly correlated with microvascular and liver capsule invasion of hepatocellular carcinoma. Functionally, knockdown of DANCR inhibited hepatocellular carcinoma cell proliferation and invasion by suppressing $\beta$-catenin signaling [16]. Silencing DANCR expression could promote hepatocellular carcinoma cell apoptosis, meanwhile cell cycle progression was blocked in G1 phase. DANCR could contribute to hepatocellular carcinoma malignancy via sponging miR-216a-5p and modulating KLF12 [17]. In addition, Guo et al. reported that DANCR could decoy miR-27a-3p to regulate ROCK1/LIMK1/COFILIN1 pathway to promote hepatocellular carcinoma progression [18].

\section{Gastric cancer}

Hao et al. confirmed that DANCR was dramatically upregulated in gastric cancer tissues and DANCR overexpression was significantly associated with worse overall survival in gastric cancer patients. Their experiments revealed that overexpression of DANCR notably increased gastric cancer cell proliferation by influencing the gene expression programs in cell metabolic and cycle process [19]. DANCR overexpression was significantly correlated with lymph node metastasis and late clinical stage. LncRNA-LET was a target gene of DANCR, and DANCR could associate with enhancer of zeste homolog 2 (EZH2) and HDAC3 to epigenetically silence IncRNA-LET, then regulate gastric cancer migration and invasion [20]. Otherwise, our previous research showed that the expression of DANCR was higher in the serum of gastric cancer patients. DANCR was upregulated by SALL4 and exerted its tumor-promoting roles partly through the activation of $\beta$-catenin pathway [21]. Further research showed that DANCR expression remained high in cisplatin (DDP) resistant gastric cancer tissues or cells. DANCR overexpression showed increased survival and decreased apoptosis. In addition, DANCR overexpression could upregulate the expression of MDR1 and MRP1 to accelerate the multidrug resistance (MDR) of gastric cancer [22]. 
Table 1. Clinical significance of DANCR in various tumors

\begin{tabular}{|c|c|c|c|}
\hline Cancer type & Relevant clinicopathological features & Clinical value & References \\
\hline $\begin{array}{l}\text { Hepatocellular } \\
\text { carcinoma }\end{array}$ & $\begin{array}{l}\text { Microvascular and liver capsule invasion, tumor growth, lung } \\
\text { metastasis }\end{array}$ & $\begin{array}{l}\text { Diagnostic biomarker, therapeutic target, prognostic } \\
\text { marker }\end{array}$ & {$[15,16,18]$} \\
\hline Gastric cancer & $\begin{array}{l}\text { Worse overall survival, lymph node metastasis, advanced TNM } \\
\text { stage, tumor size, invasion depth, cisplatin resistant, poor prognosis }\end{array}$ & $\begin{array}{l}\text { Diagnostic biomarker, therapeutic target, prognostic } \\
\text { marker }\end{array}$ & {$[19,21,22]$} \\
\hline Colorectal cancer & $\begin{array}{l}\text { Worse overall survival, worse disease-free survival, lymph node } \\
\text { metastasis, advanced TNM stage, histologic grade, Duckes stage, } \\
\text { poor prognosis }\end{array}$ & Therapeutic target, prognostic marker & [23-25] \\
\hline Pancreatic cancer & $\begin{array}{l}\text { Vascular invasion, advanced T stage, advanced TNM stage, lymph } \\
\text { node metastasis, lower OS rate, shorter PFS period, poor prognosis }\end{array}$ & Therapeutic target & {$[27,28]$} \\
\hline Breast cancer & Advanced TNM stage, shorter OS & $\begin{array}{l}\text { Diagnostic biomarker, therapeutic target, prognostic } \\
\text { marker }\end{array}$ & {$[30-32]$} \\
\hline Lung cancer & $\begin{array}{l}\text { Larger tumor size, advanced TNM stage, lymph node metastasis, } \\
\text { shorter postoperative survival time, poor prognosis }\end{array}$ & $\begin{array}{l}\text { Diagnostic biomarker, therapeutic target, prognostic } \\
\text { marker }\end{array}$ & {$[36,38-40]$} \\
\hline Glioma & $\begin{array}{l}\text { Malignancy, advanced tumor grade, poor prognosis, cisplatin } \\
\text { sensitivity }\end{array}$ & $\begin{array}{l}\text { Therapeutic target, biomarker for predicting cisplatin } \\
\text { sensitivity }\end{array}$ & {$[41,42,44]$} \\
\hline Osteosarcoma & Tumor size, tumor growth, lung metastasis, poor prognosis, & Therapeutic target, prognostic marker & {$[45,46]$} \\
\hline Cervical cancer & Malignant phenotype, poor prognosis & Therapeutic target & {$[49,50]$} \\
\hline Bladder cancer & $\begin{array}{l}\text { Higher histological grade, advanced TNM stage, lymph node } \\
\text { metastasis, tumor stage, poor prognosis, }\end{array}$ & Diagnostic biomarker, therapeutic target & {$[51,52]$} \\
\hline Prostate cancer & Docetaxel resistant & Target for preventing prostate cancer metastasis & [53] \\
\hline $\begin{array}{l}\text { Nasopharyngeal } \\
\text { carcinoma }\end{array}$ & $\begin{array}{l}\text { Lymph node metastasis, poor survival, hypoxia phenotype, tumor } \\
\text { size, TNM stage, radiation resistance }\end{array}$ & $\begin{array}{l}\text { Therapeutic target, prognostic marker, biomarker and } \\
\text { therapeutic target for radiation resistance }\end{array}$ & {$[55,56,58]$} \\
\hline $\begin{array}{l}\text { Papillary thyroid } \\
\text { cancer }\end{array}$ & $\mathrm{T}$ grade, $\mathrm{TNM}$ stage & Diagnostic biomarker, & {$[59]$} \\
\hline Retinoblastoma & Poor overall survival and disease-free survival & - & {$[62]$} \\
\hline
\end{tabular}

Table 2. DANCR in tissues/serum/plasma as diagnostic and prognostic biomarkers

\begin{tabular}{|c|c|c|c|c|c|c|}
\hline Cancer type & Biomarker type & Specificity & Sensitivity & AUC & Sample & References \\
\hline Hepatocellular carcinoma & Diagnostic/prognostic & $84.3 \%$ & $80.8 \%$ & 0.868 & Plasma & {$[15,16]$} \\
\hline \multirow[t]{2}{*}{ Gastric cancer } & Diagnostic/prognostic & $67.7 \%$ & $64.6 \%$ & 0.704 & Tissues & {$[19,21]$} \\
\hline & & $79.5 \%$ & $72.7 \%$ & 0.816 & Serum & \\
\hline Colorectal cancer & Prognostic & NA & NA & NA & Tissues & [23] \\
\hline Breast cancer & Diagnostic/prognostic & NA & NA & NA & Tissues & {$[30,31]$} \\
\hline \multirow[t]{2}{*}{ Lung cancer } & Diagnostic/prognostic & NA & NA & 0.927 & Tissues & {$[39,40]$} \\
\hline & & NA & NA & 0.883 & Plasma & \\
\hline Osteosarcoma & Prognostic & NA & NA & NA & Tissues & [46] \\
\hline Bladder cancer & Diagnostic & NA & NA & NA & Tissues & [51] \\
\hline Nasopharyngeal carcinoma & Diagnostic/prognostic & NA & NA & NA & Tissues & {$[55,58]$} \\
\hline \multirow[t]{3}{*}{ Papillary thyroid cancer } & Diagnostic & $66.2 \%$ & $85.3 \%$ & 0.823 & Tissues & [59] \\
\hline & & $82.2 \%$ & $81.5 \%$ & 0.876 & & \\
\hline & & $91.7 \%$ & $83.3 \%$ & 0.917 & & \\
\hline
\end{tabular}

\section{Colorectal cancer}

In 2015, Liu et al. reported DANCR expression was increased in colorectal cancer tissues compared with that in adjacent normal tissues. And high DANCR expression was correlated with TNM stage, histologic grade, and lymph node metastasis. They found that DANCR expression was an independent poor prognostic factor for both overall survival (OS) and disease-free survival (DFS) [23]. Zhao et al. found that ANCR and EZH2 were highly expressed in colorectal cancer tissues, and correlated with lymphatic metastasis, Dukes stage and TNM stage. Their results suggested that ANCR could influence the invasion and migration of colorectal cancer cells by specifically binding to EZH2 [24]. Wang et al. reported that DANCR and heat shock protein 27
(HSP27) were both targets of miR-577 and shared the same binding site. DANCR could promote HSP27 expression and its mediation of proliferation/ metastasis via miR-577 sponging [25].

\section{Pancreatic cancer}

Chen et al. revealed that DANCR could promote pancreatic ductal adenocarcinoma progression, with relatively higher expression levels in pancreatic ductal adenocarcinoma cell lines and tissues. Correlation analysis of clinicopathological features and DANCR expression found that high DANCR expression was statistically correlated with vascular invasion, advanced $\mathrm{T}$ stage, lymph node metastasis and advanced TNM stage. High DANCR expression was identified as an independent risk factor for poor OS and progression-free survival (PFS) of pancreatic 
ductal adenocarcinoma. DANCR could promote the proliferation and metastasis of pancreatic ductal adenocarcinoma cells. Besides, miR-33a-5p/ AXL signaling pathway may be involved in mediating the function of DANCR [26]. Yao et al. reported that DANCR was markedly upregulated in clinical tissues and cell lines of pancreatic cancer. High DANCR expression exhibited a significant correlation with poor prognosis. And DANCR could promote pancreatic cancer cell growth and metastasis through working as a competing endogenous RNA (ceRNA) of miR-214-5p, and positively regulate E2F2 expression in pancreatic cancer cells [27]. Otherwise, A negative relationship was found between DANCR and miR135 a expression in pancreatic cancer. DANCR was downregulated by miR-135a through regulating of downstream protein NLRP3 in pancreatic cancer [28].

\section{Breast cancer}

Li et al. initially identified that ANCR could potentiate the CDK1-EZH2 interaction, which then increase the intensity of phosphorylation at Thr-345 and Thr-487 sites of EZH2, facilitating EZH2 ubiquitination and hence its degradation. They uncovered that ANCR level was lower in breast cancer tissues and breast cancer cell lines, in contrast to their normal counterparts. ANCR is an important player in breast cancer progression and metastasis mainly through decreasing EZH2 stability [29]. TGF- $\beta$ participates in epithelial-mesenchymal transition (EMT) and involves in physiological and pathological functions of tumor progression. Li et al. confirmed that ANCR participated in TGF- $\beta 1$-induced EMT and TGF- $\beta 1$ could down-regulate ANCR expression by increasing HDAC3 enrichment at ANCR promoter region, which decrease both $\mathrm{H} 3$ and $\mathrm{H} 4$ acetylation of ANCR promoter in breast cancer. Downstream, ANCR could inhibit breast cancer cell migration and breast cancer metastasis by decreasing RUNX2 in vitro and in vivo. In addition, ectopic expression of ANCR partly attenuated the TGF- $\beta 1$-induced EMT. Therefore, as a new TGF- $\beta$ downstream molecular, ANCR is essential for TGF- $\beta 1$-induced EMT by decreasing RUNX2 expression [30].

Table 3. Functional characterization of DANCR in various tumors

\begin{tabular}{|c|c|c|c|c|c|}
\hline Cancer types & Expression & Role & Functional role & Related genes/pathway & References \\
\hline $\begin{array}{l}\text { Hepatocellular } \\
\text { carcinoma }\end{array}$ & Overexpression & Oncogene & $\begin{array}{l}\text { Proliferation, invasion, metastasis, } \\
\text { apoptosis, cell cycle, EMT progression, } \\
\text { stemness feature, colonization }\end{array}$ & $\begin{array}{l}\beta \text {-catenin pathway, miR-216a-5p, KLF2, } \\
\text { miR-27a-3p, miR-214, miR-320a, miR-199a, } \\
\text { CTNNB1 }\end{array}$ & [15-18] \\
\hline Gastric cancer & Overexpression & Oncogene & $\begin{array}{l}\text { Proliferation, invasion, metastasis, } \\
\text { apoptosis, cell metabolic, cell cycle, EMT } \\
\text { progression, multidrug resistance }\end{array}$ & $\begin{array}{l}\text { EZH2, HDAC3, IncRNA-LET, SALL4, } \\
\beta \text {-catenin pathway, MDR1, MRP1 }\end{array}$ & [19-22] \\
\hline Colorectal cancer & Overexpression & Oncogene & Proliferation, invasion, metastasis & EZH2, H3K27me3, miR-577, HSP27 & [23-25] \\
\hline Pancreatic cancer & Overexpression & Oncogene & Proliferation, invasion, metastasis & $\begin{array}{l}\text { miR-33a-5p, AXL, miR-214-5p, E2F2, miR-135a, } \\
\text { NLRP3 }\end{array}$ & [26-28] \\
\hline \multirow[t]{2}{*}{ Breast cancer } & Overexpression & Oncogene & Proliferation, invasion, cell growth & $\begin{array}{l}\text { EZH2, CD } 44, \text { ABCG2, miR-216-5p, Nanog, } \\
\text { SOX2, Oct4, RXRA, GSK3 } \beta \text {, PIK3CA, } \\
\text { PI3K/AKT signaling pathway }\end{array}$ & [31-33] \\
\hline & Downregulation & Suppressor & Metastasis, migration, EMT progression & CDK1-EZH2, TGF- $\beta 1$, HDAC3, RUN2 & {$[29,30]$} \\
\hline \multirow[t]{2}{*}{ Lung cancer } & Overexpression & Oncogene & $\begin{array}{l}\text { Proliferation, invasion, migration, } \\
\text { apoptosis, colony formation, cell cycle, cell } \\
\text { growth, EMT progression }\end{array}$ & $\begin{array}{l}\text { miR-216a, mTOR, miR-496, HMGA2, } \\
\text { miR-758-3P, Sox4, miR-138, EZH2, p21 }\end{array}$ & [34-39] \\
\hline & Downregulation & Suppressor & Invasion, migration & TGF- $\beta 1$ & {$[40]$} \\
\hline Glioma & Overexpression & Oncogene & $\begin{array}{l}\text { Proliferation, invasion, metastasis, } \\
\text { apoptosis, cell cycle, }\end{array}$ & $\begin{array}{l}\text { miR-33a-5p, miR-645, RAB1A, } \beta \text {-catenin } \\
\text { pathway, AXL, miR-33b-5p, miR-1-3p, } \\
\text { miR-206, miR-613, NF-kB signaling pathway }\end{array}$ & [41-44] \\
\hline Osteosarcoma & Overexpression & Oncogene & Proliferation, invasion, metastasis, & $\begin{array}{l}\text { miR-33a-5p, AXL, PI3K-AKT signaling } \\
\text { pathway, miR-335-5p, miR-1972, ROCK1, } \\
\text { EZH2, p21, p27 }\end{array}$ & [45-48] \\
\hline cervical cancer & Overexpression & Oncogene & Proliferation, invasion, metastasis & $\begin{array}{l}\text { miR-335-5p, miR-665, TGFBR1, ERK/SMAD } \\
\text { pathway }\end{array}$ & {$[49,50]$} \\
\hline Bladder cancer & Overexpression & Oncogene & $\begin{array}{l}\text { Proliferation, invasion, metastasis, } \\
\text { malignant phenotype, EMT progression }\end{array}$ & $\begin{array}{l}\text { miR-149, MSI2, IL-11-STAT3 signaling, cyclin } \\
\text { D1, PLAU, LRPPRC }\end{array}$ & {$[51,52]$} \\
\hline Prostate cancer & Overexpression & Oncogene & $\begin{array}{l}\text { Proliferation, invasion, metastasis, } \\
\text { migration, malignant phenotype, EMT } \\
\text { progression }\end{array}$ & $\begin{array}{l}\text { TIM2/3, EZH2, androgen-AR signaling } \\
\text { pathway, miR-34a-5p, JAG1 }\end{array}$ & {$[53,54]$} \\
\hline $\begin{array}{l}\text { Nasopharyngeal } \\
\text { carcinoma }\end{array}$ & Overexpression & Oncogene & $\begin{array}{l}\text { Proliferation, invasion, metastasis, } \\
\text { migration, apoptosis, cell growth }\end{array}$ & $\begin{array}{l}\text { HIF-1a, NF90/NF45, AKT, PTEN, IL-6, STAT3, } \\
\text { JAK1, EZH2 }\end{array}$ & [55-58] \\
\hline $\begin{array}{l}\text { Esophageal squamous } \\
\text { cell carcinoma }\end{array}$ & Overexpression & Oncogene & Proliferation, invasion, migration, apoptosis & - & {$[60]$} \\
\hline Ovarian cancer & Overexpression & Oncogene & Proliferation, invasion, migration & IGF2 & {$[61]$} \\
\hline Retinoblastoma & Overexpression & Oncogene & $\begin{array}{l}\text { Proliferation, invasion, migration, EMT } \\
\text { progression }\end{array}$ & $\begin{array}{l}\text { N-cadherin, Vimentin, miR-34c, miR-613, } \\
\text { MMP-9 }\end{array}$ & {$[62]$} \\
\hline
\end{tabular}


While, Sha, Tao and Tang et al. identified that DANCR was significantly overregulated in triple negative breast cancer tissues and cell lines [31-33]. Kaplan-Meier analysis showed that higher DANCR expression was correlated with worse TNM stages and a shorter OS. Further study indicated that DANCR overexpression significantly promoted cell proliferation and invasion in vitro and contributed to tumor growth in vivo. Sha et al. revealed that DANCR knockdown was associated with increased binding of $\mathrm{EZH} 2$ on the promoters of CD44 and ABCG2, and concomitant reduction of expression of these genes, suggesting that they may be DANCR targets in triple negative breast cancer [31]. Tao et al demonstrated that miRNA-216a-5p was a target of DANCR by bioinformatics analysis. Their experiments demonstrated that miRNA-216a-5p interacted with DANCR and its inhibitor could weaken the influences induced by DANCR knockdown for cancer cells, including cell proliferation and invasion, and the expression of Nanog, SOX2 and Oct4 [32]. In addition, Tang et al. demonstrated that DANCR bound with RXRA and increased its serine $49 / 78$ phosphorylation via GSK3 $\beta$, resulting in activating PIK3CA transcription, and subsequently enhanced PI3K/AKT signaling and triple negative breast cancer tumorigenesis [33].

\section{Lung cancer}

Zhen et al. saw DANCR upregulation in lung cancer, particularly in high-grade lung cancer tissues and aggressive cancer cells. The DANCR overexpression promoted cell proliferation and colony formation in vitro, and DANCR interference effectively suppressed lung cancer progression both in vitro and in vivo. They found that the miR-216a level in cancer cells was negatively correlated with DANCR expression [34]. Lu et al. demonstrated that DANCR was up-regulated in lung adenocarcinoma and that DANCR knockdown inhibited tumor cell proliferation, migration and invasion and restored cell apoptosis rescued. They found that mTOR was a target of miR-496 and that DANCR could modulate the expression levels of mTOR by working as a ceRNA [35]. In addition, elevated DANCR expression was associated with poor prognosis in the patients with lung adenocarcinoma. And DANCR promoted the invasive ability via regulation of high mobility group AT-hook 2 (HMGA2) in lung adenocarcinoma cells, SPCA1 and A549 [36].

The study of Wang revealed that DANCR was markedly upregulated in non-small-cell lung cancer tumor tissues and cell lines compared with related normal controls. DANCR could function as a tumor promoter and promote cell proliferation, invasion and migration through directly targeting the tumor suppressor miR-758-3p in non-small-cell lung cancer [37]. The upregulation of DANCR expression was significantly associated with larger tumor size, advanced TNM stage and lymph node metastasis, and also predicted poor prognosis of patients with non-small-cell lung cancer. DANCR could compete with Sox4 mRNA to bind with miR-138, thus affecting Sox4 expression. In addition, Sox4 could bind to the promoter regions of DANCR gene to activate DANCR expression, suggesting a positive feedback loop of DANCR/miR-138/Sox4 in non-small-cell lung cancer [38]. Guo et al. revealed that the knockdown of DANCR inhibited non-small-cell lung cancer cell proliferation by inducing cell apoptosis and cell cycle arrest. In addition, DANCR knockdown suppressed non-small-cell lung cancer cell migration and invasion via inhibition of EMT. DANCR knockdown inhibited EZH2-mediated epigenetic silencing of p21 promoter and increased p21 expression [39]. While, Wang et al. found that ANCR was significantly downregulated in non-small-cell lung cancer patients compared with healthy controls in lung biopsies and plasma. Downregulated expression of ANCR distinguished non-small-cell lung cancer patients from healthy controls and low non-small-cell lung cancer expression level indicated shorter postoperative survival time of non-small-cell lung cancer patients. ANCR inhibited non-small-cell lung cancer cell migration and invasion, at least partially by downregulating TGF- $\beta 1$ expression [40].

\section{Glioma}

Yang et al. explored that DANCR was increased in glioma tissues and cells compared with normal brain tissues and cells. DANCR expression was positively correlated with the malignancy and poor prognosis of glioma patients. DANCR contained a binding site of miR-33a-5p and DANCR expression was negatively correlated with the expression of miR-33a-5p in glioma tissues. DANCR could facilitate cancer cell proliferation, invasion and metastasis and inhibit apoptosis through competitively binding to miR-33a-5p in glioma [41]. High DANCR expression was correlated with advanced tumor grade. Inhibition of DANCR suppressed the glioma cell proliferation and induced cell arrested in the G0/G1 phase. DANCR could directly interact with miR-634 in glioma cells and this interaction results in the inhibition of downstream of RAB1A expression [42]. $\mathrm{Li}$ et al. verified that DANCR positively affected glioma progression via activating $\mathrm{Wnt} / \beta$-catenin signaling pathway [43]. In addition, DANCR expression was negatively correlated with cisplatin sensitivity in glioma cells. DANCR could attenuate 
cisplatin-induced cell proliferation inhibition in vitro, xenograft growth suppression in vivo and cisplatin-induced cell apoptosis in vitro and in vivo. DANCR upregulated AXL via competitively binding miR-33a-5p, miR-33b-5p, miR-1-3p, miR-206 and miR-613. Through upregulating AXL, DANCR activated $\mathrm{PI} 3 \mathrm{~K} / \mathrm{Akt} / \mathrm{NF}-\mathrm{kB}$ signaling pathway in glioma cells. DANCR could promote cisplatin resistance via activating AXL/PI3K/Akt/ NF-kB signaling pathway in glioma [44].

\section{Osteosarcoma}

The present study of Jiang et al. showed that DANCR was significantly increased in osteosarcoma tissues, and its expression was positively correlated with tumor size and metastasis as an independent poor prognostic factor. The overexpression of DANCR could increase osteosarcoma cell proliferation, migration, and invasion in vitro, as well as promote xenograft tumor growth and lung metastasis in vivo. DANCR upregulated expression of the receptor tyrosine kinase AXL by competitively binding to miR-33a-5p, which targets AXL mRNA for degradation, and this function was sequentially performed through the PI3K-AKT signaling pathway [45]. Wang et al. firstly demonstrated that DANCR could decoy two miRNAs - miR-335-5p and miR-1972 to facilitate ROCK1-mediated proliferation and metastasis in osteosarcomab [46].

Li et al. found that ANCR was highly expressed in human osteosarcoma cell lines. And their data indicated that ANCR might be an oncogenic lncRNA that promoted proliferation of osteosarcoma [47]. ANCR depletion inhibited the proliferation, invasion, and migration of osteosarcoma cells. Downregulation of ANCR could decrease the abundance of EZH2 and activate the expression of both p21 and p27. The interaction between ANCR with EZH2 indicated that ANCR might exert its function via binding to EZH2 [48].

\section{Cervical cancer}

In Liang's study, they discovered that DANCR was significantly elevated in cervical cancer tissues and cells, and was closely correlated with poor prognosis of cervical cancer patients. In addition, knockdown of DANCR inhibited cervical cancer cell proliferation, migration, and invasion in vitro, indicating that DANCR functioned as an oncogene in cervical cancer. They verified that DANCR could regulate ROCK1 expression by competitively binding to miR-335-5p [49]. In addition, Cao et al. identified miR-665 as the ceRNA for DANCR, and their observations suggested that DANCR-mediated miR665 downregulation could regulate the malignant phenotype of cervical cancer cells by targeting transforming growth factor beta receptor 1 (TGFBR1) through the ERK/SMAD pathway [50].

\section{Bladder Cancer}

Zhao et al. found that DANCR was significantly up-regulated in bladder cancer, and increased DANCR expression was positively correlated with higher histological grade and advanced TNM stage. Knockdown of DANCR could inhibit malignant phenotypes and EMT of bladder cancer cells. And they found that DANCR was distributed mostly in the cytoplasm and functioned as a miRNA sponge to positively regulate the expression of musashi RNA binding protein 2 (MSI2) through sponging miR-149 and subsequently promoted malignant phenotypes of bladder cancer cells [51]. Chen et al discovered that DANCR was significantly upregulated in cases with lymph node metastasis. DANCR expression was positively correlated with lymph node metastasis status, tumor stage, histological grade, and poor patient prognosis. Functional assays demonstrated that DANCR promoted bladder cancer cell migration, invasion, and proliferation in vitro and enhanced tumor lymph node metastasis and growth in vivo. DANCR could activate IL-11-STAT3 signaling and increase cyclin D1 and PLAU expression via guiding leucine-rich pentatricopeptide repeat containing (LRPPRC) to stabilize mRNA [52].

\section{Prostate cancer}

In 2015, Jia et al. found the expression of DANCR was increased in prostate cancer tissues and cells compared to normal prostate cancer tissues and cells. Moreover, DANCR promoted prostate cancer cell invasion and migration in vitro and metastasis of tumor xenografts in nude mice. They found that TIMP2/3, which are critical metastasis inhibitor of prostate cancer, were down-regulated by DANCR synergistically with EZH2 through epigenetically silencing their promoter. DANCR expression was repressed by androgen-AR signaling pathway and DANCR knockdown facilitated the upregulation of TIMP2/3 and the suppression of invasion and migration by androgen-AR, while DANCR knockdown decreased the promotion of invasion and migration in prostate cancer cells by enzalutamide treatment [53]. Ma et al. reported that DANCR was significantly upregulated in docetaxel (DTX) resistant prostate cancer. Silencing of DANCR could improve the DTX efficacy in DTX-resistant prostate cancer cells. DANCR served as a ceRNA of miR-34a-5p, leading to the derepression of miR-34a-5p target JAG1 to enhance DTX resistance of prostate cancer [54]. 


\section{Nasopharyngeal carcinoma}

Wen et al. reported that DANCR was upregulated in nasopharyngeal carcinoma, especially in those with lymph lode metastasis, and its upregulation could predict poor survival. And DANCR was responsible for nasopharyngeal carcinoma metastasis and hypoxia phenotype. DANCR could stabilize HIF-1a mRNA through interacting with NF90/NF45 complex, leading to nasopharyngeal carcinoma metastasis and invasion [55]. Hao's study suggested that DANCR overexpression promoted nasopharyngeal carcinoma cell proliferation and migration and inhibited apoptosis. DANCR could regulate the phosphorylation of AKT serine/threonine kinase and the protein expression of PTEN in nasopharyngeal carcinoma cells [56]. In addition, Zhang et al. identified that DANCR was highly expressed in nasopharyngeal carcinoma cells and IL-6 stimulation upregulated DANCR expression and invasion of nasopharyngeal carcinoma cells. Their study demonstrated that DANCR, acting as an oncogene in nasopharyngeal carcinoma, promoted nasopharyngeal carcinoma progression by interacting with STAT3 and enhancing JAK1 binding to STAT3 to strengthen IL-6/JAK1/STAT3 signaling[57]. Ma et al. identified that ANCR was upregulated in nasopharyngeal carcinoma tissues and cells. ANCR expression was significantly correlated with tumor size and TNM stage. ANCR could promote nasopharyngeal carcinoma cell growth and radiation resistance by repressing the expression of PTEN. And this regulation relied on ANCR-mediated EZH2 binding and epigenetic regulation on PTEN promoter [58].

\section{Other cancers}

In recent days, some studies also reported that DANCR played important role in other cancers such as papillary thyroid cancer, esophageal squamous cell carcinoma, ovarian cancer and retinoblastoma.

Zhang et al. demonstrated that the expression of DANCR was notably decreased in papillary thyroid cancer tissues in comparison with adjacent normal tissues. DANCR expression level was correlated to T grade and TNM stage. Their results suggested that DANCR was an independent factor for TNM stage of papillary thyroid cancer [59]. Shi et al. demonstrated that the expression of DANCR in esophageal squamous cell carcinoma tissues was significantly higher compared with that in the adjacent normal tissues. Furthermore, cell proliferation, migration and invasion were significantly suppressed, while cell apoptosis was promoted by knockdown mediated downregulation of DANCR expression [60]. Gao et al. revealed that the expression of DANCR in ovarian cancer samples was significantly higher than that of the corresponding normal tissues. DANCR could enhance the proliferation, migration and invasion capacities of ovarian cancer cells by upregulating insulin-like growth factor 2 (IGF2) [61]. Wang et al. revealed that DANCR was upregulated in retinoblastoma tissues and cell lines. The ectopic overexpression of DANCR indicated poor overall survivals and DFS for retinoblastoma patients. DANCR could function as ceRNA for miR-34c and miR-613 to modulate progression and metastasis in retinoblastoma oncogenesis via targeting MMP-9 [62].

\section{Regulatory mechanisms of DANCR in human cancers}

DANCR abnormal expression in various human cancers has been shown to play active roles through regulating cancer-related phenotypes, as promoting cell proliferation, migration, invasion, inhibiting apoptosis. Furthermore, the regulatory mechanisms of DANCR are complex and involve multiple factors (Figs. 1-4).

\section{Acting as a ceRNA}

CeRNAs is a novel regulatory mechanism whereby lncRNAs function as ceRNAs to sponge microRNAs and simultaneously target both noncoding RNAs and genes [63, 64]. Several articles have confirmed that DANCR could function as a ceRNA to regulate the expression of specific genes, exerting its oncogenic function in various cancers (Fig. 1). Wang and Tao et al. demonstrated that DANCR could directly interact with miR-216a-5p to regulate the expression of KLF12, Nanog, OCT4 and SOX2 [17, 32]. Guo et al. found that DANCR could promote hepatocellular carcinoma development by decoying miR-27a-3p to regulate ROCK1/LIMK1/ COFILIN pathway [18]. Wang et al. revealed that DANCR could promote HSP27 expression and its mediation of proliferation/metastasis via miR-577 sponging in colorectal cancer [25]. The DANCR overexpression promoted cell proliferation, colony formation, migration and invasion by interacting with miR-216a and miR-758-3p and modulated mTOR expression through directly binding to miR-496 in lung cancer [34, 35, 37]. Yao et al. revealed that DANCR could positively regulate E2F2 expression through working as a ceRNA of miR-214-5p in prostate cancer cells [27]. And DANCR regulated the expression of E-cadherin, N-cadherin and NLRP3 by targeting miR-135a-5p in prostate cancer [28]. DANCR could facilitate glioma malignancy and upregulate AXL to increase osteosarcoma cells function via competitively binding to miR-33a-5p [41, 
45]. Xu et al. showed that DANCR directly interacted with miR-634 and this interaction inhibited the downstream of RAB1A expression in glioma [42]. Wang et al. and Liang et al. verified that DANCR could regulate ROCK1 expression by competitively binding to miR-335-5p [46, 49]. Cao et al. proved that DANCR could directly interact with miR-665 to regulate proliferation and metastasis of cervical cancer through the ERK/SMAD pathway [50]. Zhan et al. found that DANCR positively regulated the expression of MSI2 though sponging miR-149 and subsequently promoted malignant phenotypes of breast cancer cells [51]. In addition, Wang et al. revealed that DANCR could upregulate the MMP-9 protein expression by competitively binding to miR-34c and miR-613 [62].

\section{Epigenetic regulation}

EZH2 is one component of Polycomb Repressive Complex (PRC2), which catalyzes the trimethylation of Lys-27 of histone H3 (H3K27me3) to suppress the transcription of target genes. EZH2, as a key epigenetic regulator, participates in a variety of cancer metastasis [29]. Current researches have demonstrated that DANCR could interact with EZH2 to epigenetically silence CD44, ABCG2, p21 and TIMP2/3 expression [31, 39, 53]. Mao et al. revealed that DANCR could function as a molecular scaffold of EZH2 and HDAC3 to epigenetically silence another lncRNA, IncRNA-LET [20]. ANCR could specifically bind to EZH2 and activate p21, p27 expression in osteosarcoma and influence the invasion and migration of colorectal cancer cells [24, 48]. Li et al indicated that ANCR-EZH2 binding might facilitate
CDK1 interaction with EZH2 to promote phosphorylation at Thr-345 and Thr-487 residues of $\mathrm{EZH} 2$, and finally potentiate EZH2 ubiquitination degradation through ubiquitin-proteasome pathway [29] (Fig. 2).

\section{Participation in signaling pathways}

\section{$\beta$-catenin signaling pathway}

Previous reports indicated that the activation of $\beta$-catenin pathway promoted tumor cell proliferation and metastasis [16]. For hepatocellular carcinoma, recently, Yuan et al. indicated that DANCR increased stemness feature of hepatocellular carcinoma via derepression of catenin beta 1 (CTNNB1) [15]. Ma et al. found that the activity of $\beta$-catenin was significantly reduced by siDANCR and genes downstream of $\beta$-catenin, namely vimentin, CCND1 and MYC were all downregulated by siDANCR in hepatocellular carcinoma cells [16]. Our previous study found that DANCR knockdown decreased while DANCR overexpression increased the expression of $\beta$-catenin in gastric cancer cells. And the increased expression of $\beta$-catenin and its target gene c-Myc by DANCR was inhibited by $\beta$-catenin siRNA in gastric cancer cells. DANCR could activate $\beta$-catenin to promote gastric cancer progression [21]. In addition, western blot assay was conducted in glioma cells to demonstrate that the protein levels of the $W n t / \beta$-catenin pathway related proteins were negatively modulated by si-DANCR [43]. These findings suggest that DANCR could activate the $\beta$-catenin signaling pathway to act oncogenic roles in various cancers (Fig. 3).

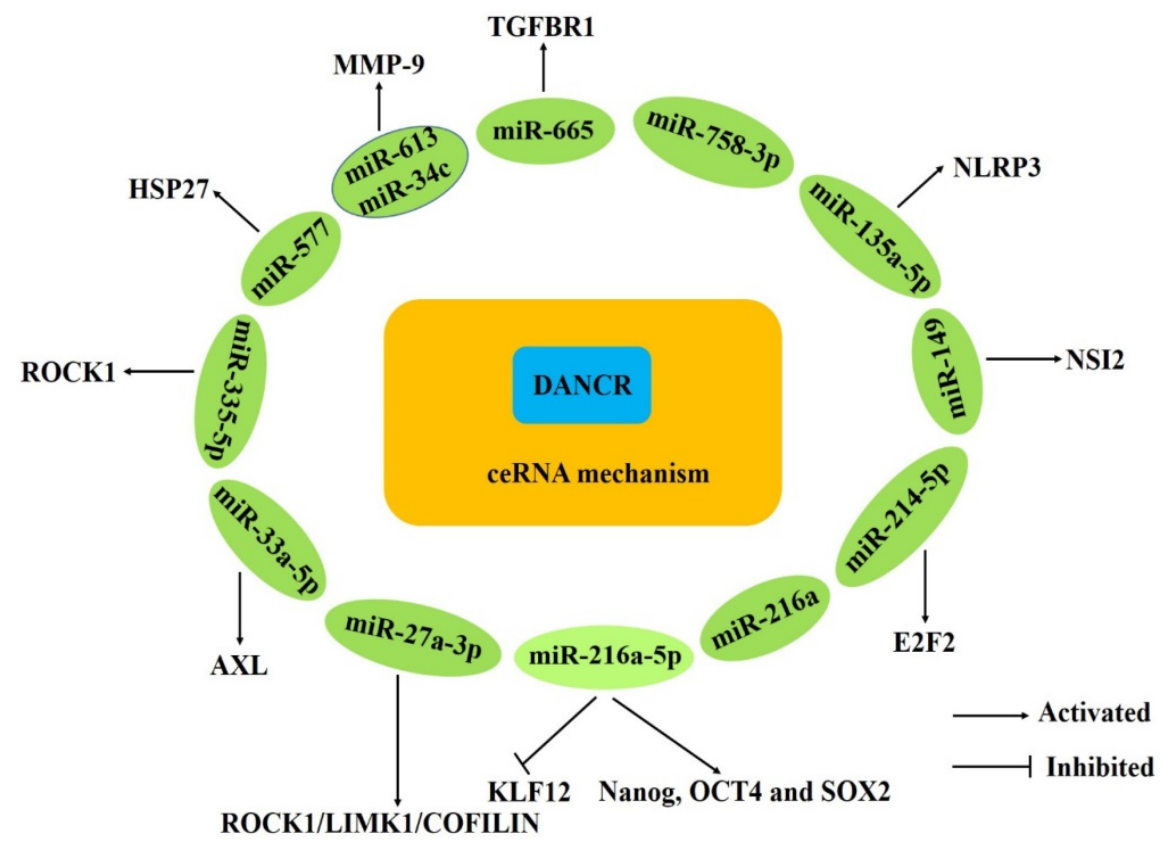

Figure 1. DANCR acts as ceRNAs in human cancers. 


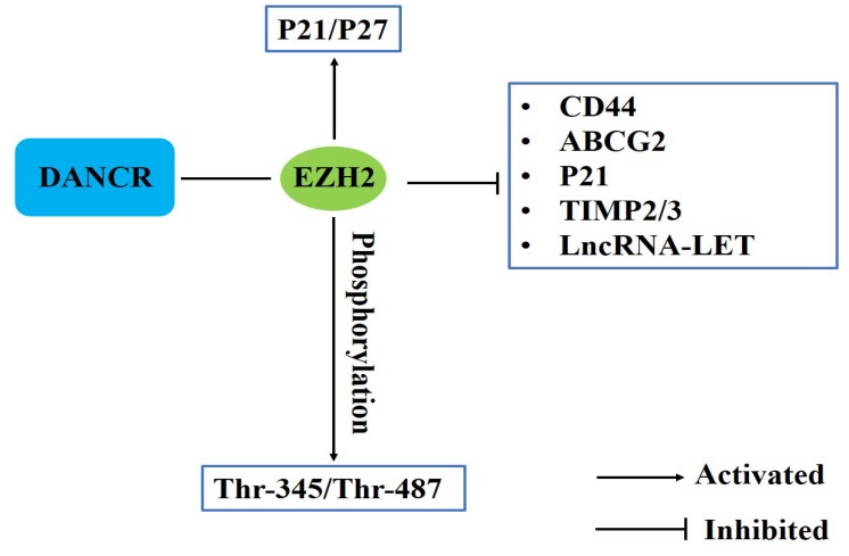

Figure 2. DANCR involves in epigenetic regulation in human cancers.

\section{PI3K signaling pathway}

The PI3K pathway plays a key role in the regulation of multiple cellular events, including cell growth, proliferation, cell cycle progression and survival [65]. Tang et al. showed that DANCR directly bound with RXRA and mediated its phosphorylation. RXRA could bind with the promoter of PIK3CA and downregulate PIK3CA transcription. DANCR knockdown inhibited RXRA protein phosphorylation and PIK3CA expression level. DANCR-induced RXRA phosphorylation suppressed RXRA-inhibited PIK3CA transcription in triple negative breast cancer cells and ultimately activated the downstream PI3K/AKT signaling [33]. AXL is an important receptor tyrosine kinase, which activates PI3K/AKT/ NF-kB signaling pathway. Ma et al. found that through upregulating AXL, DANCR increased phosphorylation levels of AKT and IKBa, and activated PI3K/AKT/NF-KB signaling pathway [44]. These findings suggest that DANCR could promote cancer progression and mediate drug resistance by activating PI3K/AKT signaling pathway (Fig. 3).

\section{TGF- $\beta$ signaling pathway}

TGF- $\beta$ signaling pathway interacts with multiple signaling pathways to participate in the development and progression of different types of human malignancies [66]. TGF- $\beta$ acts as a tumor suppressor in the early stage of tumor development by inhibiting cell proliferation and inducing EMT to promote tumor cell invasion $[67,68]$. Li et al. revealed that TGF- $\beta 1$ down-regulated ANCR expression by increasing HDAC3 enrichment at ANCR promoter region, which decreased both $\mathrm{H} 3$ and $\mathrm{H} 4$ acetylation of ANCR promoter. Western blot and transwell assays indicated that ectopic expression of ANCR partly attenuated the TGF- $\beta 1$-induced EMT [30]. Wang et al. indicated that ANCR overexpression could inhibit non-small-cell lung cancer cell migration and invasion and downregulated TGF- $\beta 1$ expression, while TGF- $\beta 1$ treatment showed no significant effects on ANCR expression but promoted non-small-cell lung cancer cell migration and invasion. TGF- $\beta 1$ treatment could attenuate the inhibitory effects of ANCR overexpression on non-small-cell lung cancer cell migration and invasion [40] (Fig. 3).

\section{Other mechanisms}

$\mathrm{Xu}$ et al. found that DANCR overexpression markedly upregulated mRNA and protein levels of MDR-related genes, such as MDR1 and MRP1 to contribute to the development of MDR [22]. Zhang et al. revealed that DANCR knockdown inhibited HMGA2 expression and the inhibition of HMGA2 suppressed the invasive ability of lung adenocarcinoma cells. DANCR could promote the invasion of lung adenocarcinoma cells by positively regulating HMGA2 [36]. LRPPRC is an RNA-binding protein that regulates mRNA stability and polyadenylation [52]. DANCR could directly interact with LRPPRC, LI-11, PLAU and CCND1 mRNA.

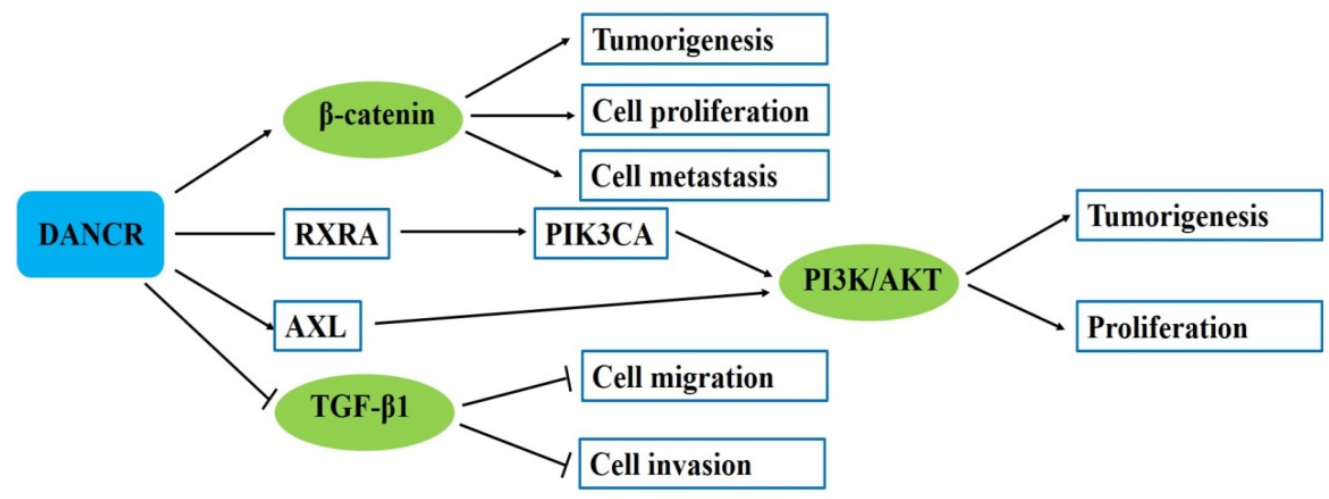

$\longrightarrow$ Activated

$\longrightarrow$ Inhibited

Figure 3. DANCR involves in several signaling pathways in human cancers. 
DANCR guided LRPPRC to stabilize IL-11, PLAU, and CCND1 mRNAs, which activated IL-11-STAT3 signaling and enhances CCND1 and PLAU expression levels [52]. Zhang et al. revealed that IL-6 promoted DANCR transcription partly by activating STAT3 phosphorylation. Besides, DANCR strengthened IL-6 stimulation-induced STAT3 phosphorylation by interacting with STAT3. There is an obviously existing positive feedback between STAT3 phosphorylation and DANCR transcription. DANCR could promote STAT3 activation by facilitating JAK1 to phosphorylate STAT3. DANCR could interact with STAT3 and enhance JAK1 binding to STAT3 to strengthen IL-6/JAK1/STAT3 signaling [57]. NF90, as a double-stranded RNA-binding protein, is transcribed from the interleukin enhancer binding factor 3 (ILF3) gene. Studies demonstrated that the NF90/NF45 complex can regulate gene expression and mRNA stability and play an important role in RNA metabolism and viral replication. DANCR could stabilize HIF-1a mRNA through interacting with NF90/NF45 complex, leading to nasopharyngeal carcinoma metastasis [55] (Fig. 4).

\section{Conclusions and Future perspectives}

With the repaid development of next-generation sequencing technology, lncRNAs have been identified dysregulated as oncogenes or tumor suppressor genes, which play important regulatory roles in tumor formation and development. Since its discovery, DANCR has been widely investigated in various human malignancies. DANCR is overexpressed and plays an oncogenic role in most types of tumors, including breast cancer and non-small-cell lung cancer. But $\mathrm{Li}$ et al. found that ANCR was downregulated in breast cancer and in particular, they demonstrated that a variable ANCR expression pattern and biological function in different cancer types and different cell lines [29]. Besides, Wang et al. suggested that ANCR was downregulated in non-small-cell lung cancer [40]. Whether the expression of DANCR is increased or decreased in breast cancer and lung cancer remains to be further detected. DANCR expression in different subtypes of breast cancer and lung cancer might be different. Amplified DANCR is dramatically correlated with multiple clinicopathological features and prognosis, such as TNM stage, lymph node metastasis and over survival. Furthermore, DANCR is included in cellular functions such as cell proliferation, migration, invasion and cell apoptosis, suggesting that DANCR contributes to tumorigenesis and tumor progression. $\mathrm{Li}$ and Wang et al. manifested that ANCR could function as a tumor suppressor [30,40]. ANCR was likely to be involved in the metastasis but not the growth of non-small-cell lung cancer [40]. The expression of ANCR might be related to different phenotypes of cancer cells. The functional roles of DANCR in different cancers might be further verified. The underlying molecular mechanisms of DANCR involved in multiple cancers have been explored preliminarily. DANCR could function as ceRNA in the regulation of specific gene expression by competing for specific miRNAs and interact with some proteins to regulate gene expression in epigenetic regulation. And DANCR is demonstrated to take part in several signaling pathways contributing to carcinogenesis and cancer progression, including $\beta$-catenin signaling pathway, PI3K signaling pathway and TGF- $\beta$ signaling pathway.

The molecular functions and cellular mechanisms by which DANCR mediates its actions are complicated and associated with multiple factors. Several experiments have been identified that lncRNAs play a role in epigenetic, transcriptional, and

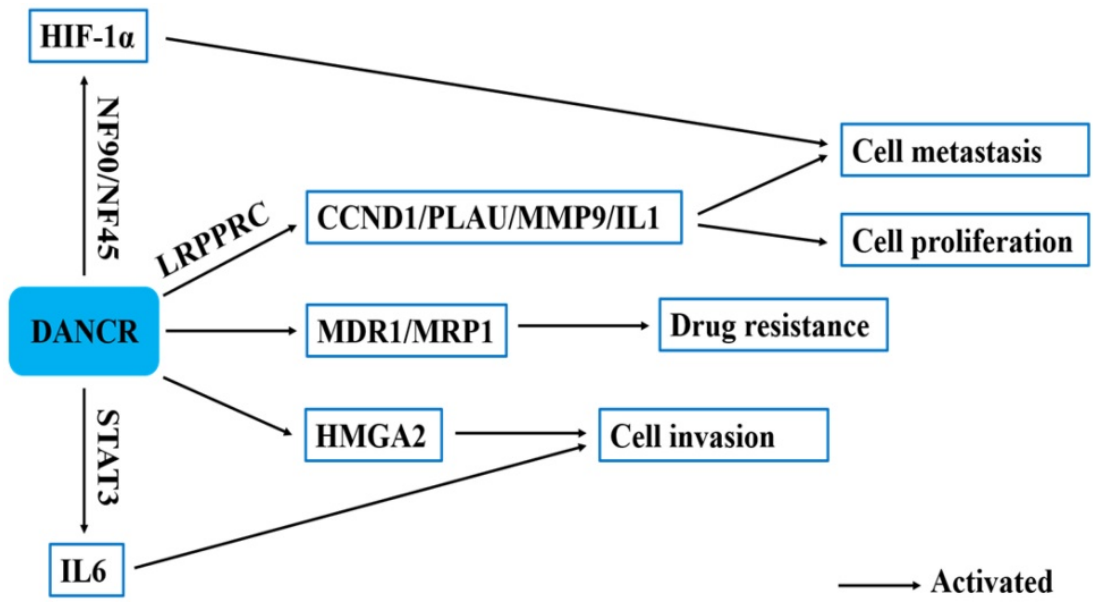

Figure 4. The other regulatory mechanisms of in human cancers. 
post-transcriptional levels to contribute carcinogenesis and cancer development. Therefore, the molecular mechanism of DANCR in each cancer type should be clarified. Recently, lncRNAs have been demonstrated not only in tumor tissues but also in body fluids including plasma, serum, urine, and so on. The expression of DANCR in body fluids including plasma should be detected. And multiple effects between DANCR and molecular target markers should be explored in most available diagnostic samples like blood to apply it in clinical use. Further studies on the clinical value of DANCR in the diagnosis and treatment of cancers require more attention.

\section{Abbreviations}

LncRNAs: long noncoding RNAs; MALAT1: metastasis-associated lung adenocarcinoma transcript 1; NKILA: NF-KappaB interacting lncRNA; DANCR: differentiation antagonizing non-protein coding RNA; ANCR: anti-differentiation non-coding RNA; TNM: tumor-node-metastasis; EZH2: enhancer of zeste homolog 2; DDP: cisplatin; MDR: multidrug resistance; OS: overall survival; DFS: disease-free survival; HSP27: heat shock protein 27; PFS: progression-free survival; ceRNA: competing endogenous RNA; HMGA2: high mobility group AT-hook 2; EMT: epithelial-mesenchymal transition; TGFBR1: transforming growth factor beta receptor 1 ; MSI2: musashi RNA binding protein 2; LRPPRC: leucine-rich pentatricopeptide repeat containing; DTX: docetaxel; IGF2: insulin-like growth factor; PRC2: Polycomb Repressive Complex; H3K27me3: the trimethylation of Lys-27 of histone H3; CTNNB1: catenin beta 1; ILF3: interleukin enhancer binding factor 3 .

\section{Acknowledgements}

We are grateful to all authors whose names are not listed but are participating in the program. This study was supported by grants from the Zhenjiang Social Development Guidance Project (FZ2018035) and the Zhenjiang Key Research and Development Project (SH2019078).

\section{Competing Interests}

The authors have declared that no competing interest exists.

\section{References}

1. Schmitt AM, Chang HY. Long noncoding RNAs in cancer pathways. Cancer Cell. 2016; 29: 452-63.

2. Huarte M. The emerging role of lncRNAs in cancer. Nat Med. 2015; 21: 1253-61.

3. Xiong T, Li J, Chen F, Zhang F. PCAT-1: a novel oncogenic long non-coding RNA in human cancers. Int J Biol Sci. 2019; 15: 847-56.
4. Guttman M, Amit I, Garber M, French C, Lin MF, Feldser D, et al. Chromatin signature reveals over a thousand highly conserved large non-coding RNAs in mammals. Nature. 2009; 458: 223-7.

5. Liu S, Qiu J, He G, Liang Y, Wang L, Liu C, et al. LncRNA MALAT1 acts as a miR-125a-3p sponge to regulate FOXM1 expression and promote hepatocellular carcinoma progression. J Cancer. 2019; 10: 6649-59.

6. Xie H, Liao X, Chen Z, Fang Y, He A, Zhong Y, et al. LncRNA MALAT1 inhibits apoptosis and promotes invasion by antagonizing miR-125b in bladder cancer cells. J Cancer. 2017; 8: 3803-11.

7. Zhang ZC, Tang C, Dong Y, Zhang J, Yuan T, Li XL. Targeting lncRNAMALAT1 suppresses the progression of osteosarcoma by altering the expression and localization of beta-catenin. J Cancer. 2018; 9: 71-80.

8. Dijkstra JM, Alexander DB. The " NF-k B interacting long noncoding RNA" ( NKILA) transcript is antisense to cancer-associated gene PMEPA1. F1000Res. 2015; 4: 96.

9. Huang W, Cui X, Chen J, Feng Y, Song E, Li J, et al. Long non-coding RNA NKILA inhibits migration and invasion of tongue squamous cell carcinoma cells via suppressing epithelial-mesenchymal transition. Oncotarget. 2016; 7: 62520-32.

10. Yu X, Tang W, Yang $\mathrm{Y}$, Tang L, Dai R, Pu B, et al. Long noncoding RNA NKILA enhances the anti-cancer effects of baicalein in hepatocellular carcinoma via the regulation of NF-kappaB signaling. Chem Biol Interact. 2018; 285: 48-58.

11. Esteller M. Non-coding RNAs in human disease. Nat Rev Genet. 2011; 12: 861-74.

12. Sanchez $Y$, Huarte M. Long non-coding RNAs: challenges for diagnosis and therapies. Nucleic Acid Ther. 2013; 23: 15-20.

13. Ling H, Vincent K, Pichler M, Fodde R, Berindan-Neagoe I, Slack FJ, et al. Junk DNA and the long non-coding RNA twist in cancer genetics. Oncogene. 2015; 34: 5003-11.

14. Kretz M, Webster DE, Flockhart RJ, Lee CS, Zehnder A, Lopez-Pajares V, et al. Suppression of progenitor differentiation requires the long noncoding RNA ANCR. Genes Dev. 2012; 26: 338-43.

15. Yuan SX, Wang J, Yang F, Tao OF, Zhang J, Wang LL, et al. Long noncoding RNA DANCR increases stemness features of hepatocellular carcinoma by derepression of CTNNB1. Hepatology. 2016; 63: 499-511.

16. Ma X, Wang X, Yang C, Wang Z, Han B, Wu L, et al. DANCR acts as a diagnostic biomarker and promotes tumor growth and metastasis in hepatocellular carcinoma. Anticancer Res. 2016; 36: 6389-98.

17. Wang J, Pu J, Zhang Y, Yao T, Luo Z, Li W, et al. DANCR contributed to hepatocellular carcinoma malignancy via sponging miR-216a-5p and modulating KLF12. J Cell Physiol. 2019; 234: 9408-16.

18. Guo D, Li Y, Chen Y, Zhang D, Wang X, Lu G, et al. DANCR promotes HCC progression and regulates EMT by sponging miR-27a-3p via ROCK1/LIMK1/ COFILIN1 pathway. Cell Prolif. 2019; 52: e12628.

19. Hao YP, Qiu JH, Zhang DB, Yu CG. Long non-coding RNA DANCR, a prognostic indicator, promotes cell growth and tumorigenicity in gastric cancer. Tumor Biology. 2017; 39: 101042831769979.

20. Mao Z, Li H, Du B, Cui K, Xing Y, Zhao X, et al. LncRNA DANCR promotes migration and invasion through suppression of lncRNA-LET in gastric cancer cells. Bioscience Reports. 2017; 37: BSR20171070.

21. Pan L, Liang W, Gu J, Zang X, Huang Z, Shi H, et al. Long noncoding RNA DANCR is activated by SALL4 and promotes the proliferation and invasion of gastric cancer cells. Oncotarget. 2018; 9: 1915-30.

22. $\mathrm{Xu}$ YD, Shang $\mathrm{J}$, Li M, Zhang YY. LncRNA DANCR accelerates the development of multidrug resistance of gastric cancer. Eur Rev Med Pharmacol Sci. 2019; 23: 2794-802.

23. Liu Y, Zhang M, Liang L, Li J, Chen YX. Over-expression of IncRNA DANCR is associated with advanced tumor progression and poor prognosis in patients with colorectal cancer. Int J Clin Exp Pathol. 2015; 8: 11480-4.

24. Yang ZY, Yang F, Zhang YL, Liu B, Wang M, Hong X, et al. LncRNA-ANCR down-regulation suppresses invasion and migration of colorectal cancer cells by regulating EZH2 expression. Cancer Biomark. 2017; 18: 95-104.

25. Wang Y, Lu Z, Wang N, Feng J, Zhang J, Luan L, et al. Long noncoding RNA DANCR promotes colorectal cancer proliferation and metastasis via miR-577 sponging. Exp Mol Med. 2018; 50: 57.

26. Chen L, Liu J, Tang T, Zhang YC, Liu MZ, Xu LY, et al. LncRNA differentiation antagonizing nonprotein coding RNA overexpression accelerates progression and indicates poor prognosis in pancreatic ductal adenocarcinoma. Onco Targets Ther. 2018; 11: 7955-65.

27. Yao Z, Chen Q, Ni Z, Zhou L, Wang Y, Yang Y, et al. Long non-coding RNA differentiation antagonizing nonprotein coding RNA (DANCR) promotes proliferation and invasion of pancreatic cancer by sponging miR-214-5p to regulate E2F2 expression. Med Sci Monit. 2019; 25: 4544-52.

28. Tang Y, Cao G, Zhao G, Wang C, Qin Q. LncRNA differentiation antagonizing non-protein coding RNA promotes proliferation and invasion through regulating miR-135a/NLRP37 axis in pancreatic cancer. Invest New Drugs. 2020; 38: s10637019007980.

29. Li Z, Hou P, Fan D, Dong M, Ma M, Li H, et al. The degradation of EZH2 mediated by lncRNA ANCR attenuated the invasion and metastasis of breast cancer. Cell Death Differ. 2017; 24: 59-71.

30. Li Z, Dong M, Fan D, Hou P, Li H, Liu L, et al. LncRNA ANCR downregulation promotes TGF-beta-induced EMT and metastasis in breast cancer. Oncotarget. 2017; 8: 67329-43. 
31. Sha S, Yuan D, Liu Y, Han B, Zhong N. Targeting long non-coding RNA DANCR inhibits triple negative breast cancer progression. Biol Open. 2017; 6: 1310-6.

32. Tao W, Wang C, Zhu B, Zhang G, Pang D. LncRNA DANCR contributes to tumor progression via targetting miR-216a-5p in breast cancer: IncRNA DANCR contributes to tumor progression. Biosci Rep. 2019; 39: BSR20181618.

33. Tang J, Zhong G, Zhang H, Yu B, Wei F, Luo L, et al. LncRNA DANCR upregulates PI3K/AKT signaling through activating serine phosphorylation of RXRA. Cell Death Dis. 2018; 9: 1167.

34. Zhen Q, Gao LN, Wang RF, Chu WW, Zhang YX, Zhao XJ, et al. LncRNA DANCR Promotes Lung Cancer by Sequestering miR-216a. Cancer Control. 2018; 25: 1073274818769849.

35. Lu QC, Rui ZH, Guo ZL, Xie W, Shan S, Ren T. LncRNA-DANCR contributes to lung adenocarcinoma progression by sponging miR-496 to modulate mTOR expression. J Cell Mol Med. 2018; 22: 1527-37.

36. Zhang N, Jiang W. Long noncoding RNA DANCR promotes HMGA2mediated invasion in lung adenocarcinoma cells. Oncol Rep. 2019; 41: 1083-90.

37. Wang S, Jiang M. The long non-coding RNA-DANCR exerts oncogenic functions in non-small cell lung cancer via miR-758-3p. Biomed Pharmacother. 2018; 103: 94-100.

38. Bai Y, Zhang G, Chu H, Li P, Li J. The positive feedback loop of lncRNA DANCR/miR-138/Sox4 facilitates malignancy in non-small cell lung cancer. Am J Cancer Res. 2019; 9: 270-84.

39. Guo L, Gu J, Hou S, Liu D, Zhou M, Hua T, et al. Long non-coding RNA DANCR promotes the progression of non-small-cell lung cancer by inhibiting p21 expression. Onco Targets Ther. 2019; 12: 135-46.

40. Wang S, Lan F, Xia Y. LncRA ANCR inhibits non-small cell lung cancer cell migration and invasion by inactivating TGF-beta pathway. Med Sci Monit. 2018; 24: 6002-9.

41. Yang JX, Sun Y, Gao L, Meng Q, Yang BY. Long non-coding RNA DANCR facilitates glioma malignancy by sponging miR-33a-5p. Neoplasma. 2018; 65: $790-8$

42. Xu D, Yu J, Gao G, Lu G, Zhang Y, Ma P. LncRNA DANCR functions as a competing endogenous RNA to regulate RAB1A expression by sponging miR-634 in glioma. Biosci Rep. 2018; 38: BSR20171664.

43. Li J, Zhou L. Overexpression of IncRNA DANCR positively affects progression of glioma via activating Wnt/beta-catenin signaling. Biomed Pharmacother. 2018; 102: 602-7.

44. Ma Y, Zhou G, Li M, Hu D, Zhang L, Liu P, et al. Long noncoding RNA DANCR mediates cisplatin resistance in glioma cells via activating AXL/ PI3K/Akt/NF-kappaB signaling pathway. Neurochem Int. 2018; 118: 233-41.

45. Jiang N, Wang $X$, Xie X, Liao $Y$, Liu N, Liu J, et al. LncRNA DANCR promotes tumor progression and cancer stemness features in osteosarcoma by upregulating AXL via miR-33a-5p inhibition. Cancer Lett. 2017; 405: 46-55.

46. Wang $Y$, Zeng $X$, Wang $N$, Zhao $W$, Zhang $X$, Teng S, et al. Long noncoding RNA DANCR, working as a competitive endogenous RNA, promotes ROCK1-mediated proliferation and metastasis via decoying of miR-335-5p and miR-1972 in osteosarcoma. Mol Cancer. 2018; 17: 89.

47. Min L, Hong S, Duan H, Zhou Y, Zhang W, Luo Y, et al. Antidifferentiation noncoding RNA regulates the proliferation of osteosarcoma cells. Cancer Biother Radiopharm. 2016; 31: 52-7.

48. Zhang F, Peng H. LncRNA-ANCR regulates the cell growth of osteosarcoma by interacting with EZH2 and affecting the expression of p21 and p27. J Orthop Surg Res. 2017; 12: 103.

49. Liang $\mathrm{H}$, Zhang $\mathrm{C}$, Guan $\mathrm{H}$, Liu J, Cui Y. LncRNA DANCR promotes cervical cancer progression by upregulating ROCK1 via sponging miR-335-5p. J Cell Physiol. 2019; 234: 7266-78.

50. Cao L, Jin $\mathrm{H}$, Zheng $\mathrm{Y}$, Mao $\mathrm{Y}, \mathrm{Fu} \mathrm{Z}$, Li $\mathrm{X}$, et al. DANCR-mediated microRNA-665 regulates proliferation and metastasis of cervical cancer through the ERK/SMAD pathway. Cancer Sci. 2019; 110: 913-25.

51. Zhan Y, Chen Z, Li Y, He A, He S, Gong Y, et al. Long non-coding RNA DANCR promotes malignant phenotypes of bladder cancer cells by modulating the miR-149/MSI2 axis as a ceRNA. J Exp Clin Cancer Res. 2018; 37: 273.

52. Chen Z, Chen X, Xie R, Huang M, Dong W, Han J, et al. DANCR promotes metastasis and proliferation in bladder cancer cells by enhancing IL-11-STAT3 signaling and CCND1 expression. Mol Ther. 2019; 27: 326-41.

53. Jia J, Li F, Tang XS, Xu S, Gao Y, Shi Q, et al. Long noncoding RNA DANCR promotes invasion of prostate cancer through epigenetically silencing expression of TIMP2/3. Oncotarget. 2016; 7: 37868-81.

54. Ma Y, Fan B, Ren Z, Liu B, Wang Y. Long noncoding RNA DANCR contributes to docetaxel resistance in prostate cancer through targeting the miR-34a-5p/JAG1 pathway. Onco Targets Ther. 2019; 12: 5485-97.

55. Wen X, Liu X, Mao YP, Yang XJ, Wang YQ, Zhang PP, et al. Long non-coding RNA DANCR stabilizes HIF-1alpha and promotes metastasis by interacting with NF90/NF45 complex in nasopharyngeal carcinoma. Theranostics. 2018; 8: 5676-89.

56. Hao $\mathrm{Y}$, Zhao H, Jin $\mathrm{X}, \mathrm{He} \mathrm{P}$, Zhang J, Dong $\mathrm{Q}$, et al. Long noncoding RNA DANCR promotes nasopharyngeal carcinoma cell proliferation and migration. Mol Med Rep. 2019; 19: 2883-9.

57. Zhang X, Yang J, Bian Z, Shi D, Cao Z. Long noncoding RNA DANCR promotes nasopharyngeal carcinoma progression by interacting with STAT3, enhancing IL-6/JAK1/STAT3 signaling. Biomed Pharmacother. 2019; 113: 108713 .
58. Ma X, Zhou J, Liu J, Wu G, Yu Y, Zhu H, et al. LncRNA ANCR promotes proliferation and radiation resistance of nasopharyngeal carcinoma by inhibiting PTEN expression. Onco Targets Ther. 2018; 11: 8399-408.

59. Zhang K, Lv J, Peng X, Liu J, Li C, Li J, et al. Down-regulation of DANCR acts as a potential biomarker for papillary thyroid cancer diagnosis. Biosci Rep. 2019; 39: BSR20181616.

60. Shi H, Shi J, Zhang Y, Guan C, Zhu J, Wang F, et al. Long non-coding RNA DANCR promotes cell proliferation, migration, invasion and resistance to apoptosis in esophageal cancer. J Thorac Dis. 2018; 10: 2573-82.

61. Gao YQ, Cheng HY, Liu KF. Long non-coding RNA DANCR upregulates IGF2 expression and promotes ovarian cancer progression. Eur Rev Med Pharmacol Sci. 2019; 23: 3621-6.

62. Wang JX, Yang Y, Li K. Long noncoding RNA DANCR aggravates retinoblastoma through miR-34c and miR-613 by targeting MMP-9. J Cell Physiol. 2018; 233: 6986-95.

63. He A, He S, Li X, Zhou L. ZFAS1: A novel vital oncogenic lncRNA in multiple human cancers. Cell Prolif. 2019; 52: e12513.

64. Dong D, Mu Z, Zhao C, Sun M. ZFAS1: a novel tumor-related long non-coding RNA. Cancer Cell Int. 2018; 18: 125.

65. Elkabets M, Pazarentzos E, Juric D, Sheng Q, Pelossof RA, Brook S, et al. AXL mediates resistance to PI3Kalpha inhibition by activating the EGFR/PKC/ mTOR axis in head and neck and esophageal squamous cell carcinomas. Cancer Cell. 2015; 27: 533-46.

66. Derynck R, Akhurst RJ, Balmain A. TGF-beta signaling in tumor suppression and cancer progression. Nat Genet. 2001; 29: 117-29.

67. Lei Z, Xu G, Wang L, Yang H, Liu X, Zhao J, et al. MiR-142-3p represses TGFbeta-induced growth inhibition through repression of TGFbetaR1 in non-small cell lung cancer. FASEB J. 2014; 28: 2696-704.

68. Wendt MK, Tian M, Schiemann WP. Deconstructing the mechanisms and consequences of TGF-beta-induced EMT during cancer progression. Cell Tissue Res. 2012; 347: 85-101. 